\title{
A review of the use of inhaled nitric oxide in the PICU at Red Cross War Memorial Children's Hospital, 2011-2015: A retrospective cohort study
}

\author{
S Padayachee, ${ }^{1}$ MB ChB, DCH, MMed (Paeds), FCPaeds (SA); OrcID 0000-0002-2428-3282 \\ S Salie, ${ }^{1,2}$ MB ChB, DCH, FCPaeds (SA), Cert Crit Care (Paed), MPH; OrcID 000-0002-8368-6321 \\ ${ }^{1}$ Department of Paediatrics and Child Health, Faculty of Health Sciences, University of Cape Town, South Africa \\ ${ }^{2}$ Paediatric Intensive Care Unit, Red Cross War Memorial Children's Hospital, Cape Town, South Africa
}

Corresponding author: S Padayachee (sandhia1@live.com)

\begin{abstract}
Background. Inhaled nitric oxide (iNO) functions as a selective pulmonary vasodilator. It is an expensive treatment that is often employed as rescue therapy for refractory hypoxaemia in acute respiratory distress syndrome (ARDS) and pulmonary hypertension (PHT) following cardiac surgery. Objectives. To describe the use of iNO and the cost of treatment in our paediatric intensive care unit (PICU).

Methods. A retrospective descriptive study of all patients treated with iNO in the PICU at Red Cross War Memorial Children's Hospital (RCWMCH) from 2011 - 2015.

Results. We treated 140 patients with iNO, 82 for PHT following cardiac surgery, 53 for ARDS and 5 for persistent pulmonary hypertension of the newborn (PPHN). A response to treatment was observed in $64 \%$ of the cohort as a whole, $80 \%$ of those with PPHN, $67 \%$ of those with PHT post-cardiac surgery, and 64\% of those with ARDS. A longer duration of PICU and hospital admission, and higher in-hospital mortality (53\%), was seen in the group with ARDS, in particular those with adenoviral infection (63\%), when compared with patients treated for PHT post-cardiac surgery (18\%) and for PPHN (20\%). The total cost of treatment with iNO was ZAR1 441376 for the 5-year period studied. There are no protocols guiding the use of iNO in our unit, and it was found that response to treatment was not being objectively measured and documented, and that practice varied between clinicians.

Conclusions. Considering the cost of treatment and lack of evidence showing improved outcomes with iNO therapy, its continued use in our resource-limited setting should be guided by protocol.
\end{abstract}

Keywords. nitric oxide; iNO; pulmonary hypertension; children; PICU.

South Afr J Crit Care 2021:37(2):45-49. https://doi.org/10.7196/SAJCC.2021.v37i2.416

Contributions of the study. There is a paucity of data regarding the indications for use, and outcomes of patients treated with iNO in resourcelimited settings. We did not find evidence of improved outcomes in patients treated with iNO despite the high costs of the therapy. Protocols should be developed to guide the use of iNO in resource-limited settings.

Nitric oxide (NO) has many features of an ideal pulmonary vasodilator. When inhaled, it has a rapid onset of action, is delivered directly to the lungs thereby minimising systemic side-effects, and improves ventilation-perfusion mismatch. ${ }^{[1,2]}$ It was first approved by the Food and Drug Administration (FDA) in 1999 for use in neonates with persistent pulmonary hypertension of the newborn (PPHN). Since then, there has been considerable interest in its potential role as a pulmonary vasodilator in other conditions, and it is often employed in severe, refractory hypoxaemia secondary to acute respiratory distress syndrome (ARDS), and in pulmonary hypertension (PHT) following cardiac surgery. ${ }^{[3]}$ It is an expensive treatment, and whether or not it improves outcomes remains controversial.

Inhaled nitric oxide (iNO) has been shown to only transiently improve oxygenation, ${ }^{[4-11]}$ and no improvement in survival has been demonstrated in patients with ARDS. ${ }^{[1,4-6,10-12]}$ Most studies date back as far as 15 years and very few randomised controlled trials (RCTs) have been conducted in children.
Treatment with iNO has been shown to decrease mortality in patients with PHT following cardiac surgery. ${ }^{[14,15]}$ Although some small studies have shown a decrease in the number of pulmonary hypertensive crises (PHTCs) postoperatively, ${ }^{[15]}$ pooled data show otherwise. ${ }^{[14,16]}$ There has also been no significant impact on mean pulmonary artery pressure (MPAP), mean arterial pressure (MAP) ${ }^{[14]}$ and oxygenation demonstrated. ${ }^{[14,16]}$ The American Heart Association (AHA) and the European Consensus guidelines suggest early use of iNO for its possible effect in decreasing the number of PHTCs and decreasing pulmonary vascular resistance (PVR). ${ }^{[1,17,18]}$ These guidelines were, however, developed in well-resourced settings.

There are limited published data on the use of iNO in resourcelimited settings, where extracorporeal membrane oxygenation (ECMO) is not available. It is also not known whether there are subgroups of patients with different underlying diseases or comorbidities that respond differently to treatment. In our unit, there are no protocols in place guiding its use, and the indications for use, dosage, weaning 
and discontinuation are usually left to the discretion of the attending physician.

Owing to the paucity of evidence showing improved outcomes with the use of iNO and the difference in context between our setting and that of most of the published data, we aimed to review its use and the outcomes of patients treated at a tertiary paediatric hospital in Western Cape Province, South Africa.

\section{Methods}

A retrospective, descriptive study was conducted reviewing all patients treated with iNO in the paediatric intensive care unit (PICU) at Red Cross War Memorial Children's Hospital (RCWMCH) from 2011 - 2015. The 22-bed multi-disciplinary PICU admits $~ 1300$ patients annually, including acute medical admissions, trauma patients and post-surgery patients. Approximately $60 \%$ of admissions are acute medical admissions and 320 children are admitted electively postcardiac surgery each year.

Ethical approval was obtained from the RCWMCH Departmental Research Committee, as well as the Human Research Ethics Committee of the University of Cape Town (ref. no. 369/2016)

Patients who received treatment with iNO during the period being reviewed were identified using the electronic PICU database. All patients were included.

Data pertaining to response to treatment, duration of admission to PICU, underlying diagnoses, comorbidities and patient characteristics were extracted from the PICU electronic database and patients' medical records. The duration of hospital admission and survival to discharge was obtained from the Clinicom database. Results of tests for retroviral disease, blood cultures and respiratory tract specimens were accessed via the National Health Laboratory System (NHLS). Echocardiographic evidence of pulmonary hypertension was obtained from the electronic database in the PICU as well as the department of cardiology's electronic database of echocardiograms.

Data relating to costs of using the treatment were obtained from the pharmacy at RCWMH. Data were entered into a standardised data collection sheet and transferred into a Microsoft Excel spreadsheet. Names and folder numbers were removed to maintain confidentiality and anonymity. Missing data were included and marked as unknown. Medians, proportions and interquartile ranges (IQRs) were determined, and the Fisher's exact test and Kruskall-Wallis rank tests were used to test the strength of associations. Stata software, version 12.0 (STATA Corp., USA) for Mac, was used to analyse data.

The number of patients who responded to treatment in the cohort as a whole as well as in subgroups with different underlying conditions was assessed. A positive response to treatment was defined as any subjective improvement judged by the attending clinician and documented in the medical records. This was often based on an improvement in oxygen saturation alone.

Mortality, length of treatment, length of admission as well as comorbidities present and their association with outcomes were also assessed.

\section{Results \\ Study Population}

A total of 140 patients received treatment with iNO from 2011 - 2015 at RCWMCH. The characteristics of these patients are shown in Table 1.

The majority of the patients (83\%; $n=116 / 140$ ) received treatment with iNO as rescue therapy, either during an acute PHTC following cardiac surgery or for refractory hypoxaemia thought to be related to raised pulmonary pressures in ARDS (Fig. 1). Less than a tenth of patients $(5 \% ; n=7 / 140)$ were treated prophylactically to prevent PHTC following cardiac surgery. The reason for initiation of treatment was not documented in 17 patients (Fig. 1).

All patients who were treated for ARDS had evidence of sepsis in the form of either a positive blood culture, sputum culture or viral panel on sputum. Of the children treated for ARDS, $8 \%(n=4 / 53)$ were admitted to the ICU for burns, $9 \%(n=5 / 53)$ following abdominal surgery, and 47\% $(n=25 / 53)$ had comorbid cardiac disease. Oral sildenafil was used to facilitate weaning off iNO in $47 \%(n=66 / 140)$ of patients in the cohort.

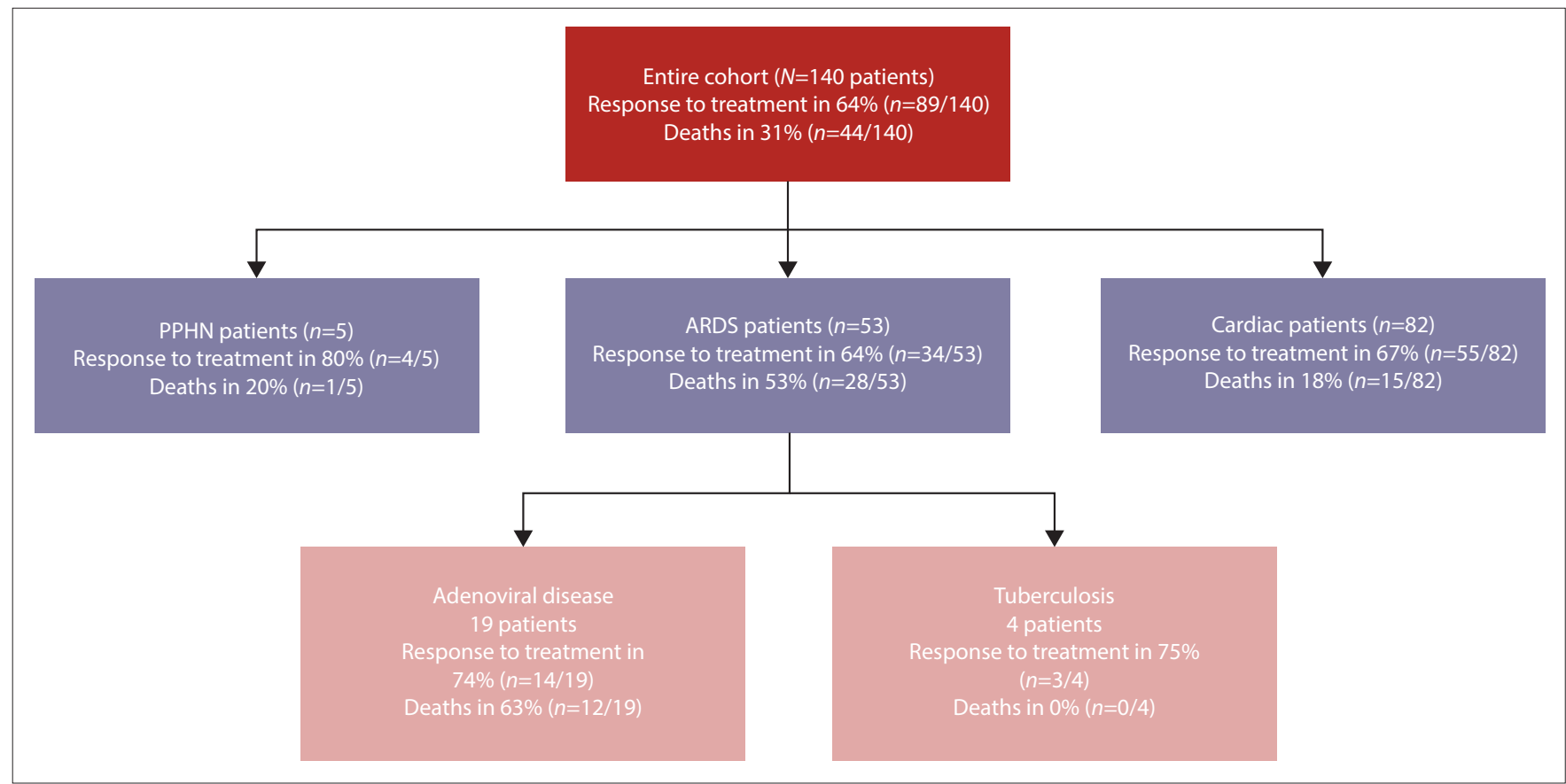

Fig. 1. Characteristics of patients treated with iNO. (PPHN = pulmonary hypertension of the newborn; $A R D S=$ acute respiratory distress syndrome; iNO $=$ inhaled nitric oxide.) 
Table 1. Patient characteristics

\begin{tabular}{ll}
\hline & $\boldsymbol{n}(\%)^{*}$ \\
\hline Male & $72(51)$ \\
Female & $68(49)$ \\
Age (months), median (IQR) & $3(1-9)$ \\
Weight (kg), median (IQR) & $4.5(3.2-8.2)$ \\
Premature at birth & \\
Underweight for age & $23(16)$ \\
Pulmonary tuberculosis & $72(51)$ \\
Sepsis & $4(3)$ \\
HIV-positive & $101(72)$ \\
IQR = interquartile range. & $5(4)$ \\
${ }^{*}$ Unless otherwise specified. \\
$\begin{array}{l}{ }^{+} \text {Gestational age }<37 \text { weeks at birth. } \\
{ }^{*} \text { Weight for age }<-2 \text { Z-score on WHO growth charts. }\end{array}$
\end{tabular}

Table 2. Deaths in ICU and in hospital inpatients treated with iNO $(N=140)$

\begin{tabular}{|c|c|c|}
\hline & $\begin{array}{l}\text { Deaths in ICU, } \\
n(\%)\end{array}$ & $\begin{array}{l}\text { Deaths in hospital, } \\
n(\%)\end{array}$ \\
\hline Entire cohort & $36(26)$ & $44(31)$ \\
\hline PHT post cardiac surgery & $9(11)$ & $15(18)$ \\
\hline ARDS & $26(49)$ & $28(53)$ \\
\hline PPHN & $1(20)$ & $1(20)$ \\
\hline Rescue & $29(25)$ & $34(29)$ \\
\hline Prophylaxis & 0 & $2(25)$ \\
\hline $\mathrm{WFA}<-2^{*}$ & $19(26)$ & $25(35)$ \\
\hline $\mathrm{WFA}>-2^{+}$ & $16(25)$ & $18(29)$ \\
\hline HIV-positive & $3(60)$ & $3(60)$ \\
\hline HIV-negative & $33(25)$ & $41(31)$ \\
\hline Premature at birth & $8(35)$ & $9(39)$ \\
\hline Sepsis & $24(24)$ & $31(31)$ \\
\hline TB & 0 & 0 \\
\hline Adenovirus & $11(58)$ & $12(63)$ \\
\hline Responders & $22(24)$ & $26(28)$ \\
\hline Non-responders & $5(25)$ & $6(30)$ \\
\hline \multicolumn{3}{|c|}{$\begin{array}{l}\text { ICU }=\text { intensive care unit; } \mathrm{iNO}=\text { inhaled nitric oxide; } \mathrm{PHT}=\text { pulmonary hypertension } \\
\text { ARDS = acute respiratory distress syndrome; PPHN = pulmonary hypertension of the } \\
\text { newborn; WFA = weight for age; TB = tuberculosis. } \\
\text { *Weight for age }<-2 Z \text {-score on WHO growth chart. } \\
\text { 'Weight for age }>-2 Z \text {-score on WHO growth chart. }\end{array}$} \\
\hline
\end{tabular}

The numbers of deaths in PICU compared with those at any point during hospital admission are shown in Table 2. Strengths of associations between patient characteristics and deaths in ICU and in hospital were tested, and patients with adenoviral disease had a significantly higher association with death in ICU $(p=0.000)$ and death in hospital $(p=0.000)$.

Patients with sepsis had longer lengths of treatment $(p=0.02)$, longer lengths of ICU admission ( $p=0.00)$, and longer lengths of hospital admission $(p=0.00)$ when associations between patient characteristics and length of treatment, and ICU and hospital days, were tested (Table 3).

\section{Cost per annum}

The total expenditure over 5 years was ZAR1 441376 and the average annual expenditure was ZAR288 275 (Table 4).

\section{Review of clinical practice}

Reviewing of patient records revealed that practice was not standardised among clinicians, and objective parameters defining a response to treatment were not used. Attending clinician used their discretion to define treatment response and this was often based on an improvement in oxygen saturation alone. Oxygenation indices and echocardiographic findings of PHT were not used routinely. It was not possible to retrospectively calculate oxygenation indices in our cohort due to missing data.

\section{Discussion}

Our cohort comprised a young population with a median age of 3 months. A positive response to treatment was seen in $64 \%$ of our cohort and was similar in those treated for ARDS and PHT following cardiac surgery. This is similar to findings by Medjo et al. ${ }^{[10]}$ and Day et al. ${ }^{[7]}$ who reported positive responses in $69 \%$ and $58 \%$ of patients treated for ARDS, respectively. Fioretto et al. ${ }^{[19]}$ reported a $94 \%$ response to treatment in patients with ARDS. This was however a small trial that probably pre-dated the change to lung protective ventilatory strategies. Our findings were limited as a standard definition of response to treatment was not used and a positive response was defined as any subjective improvement observed by the attending clinician.

We found that patients with adenoviral disease and tuberculosis demonstrated better responses to treatment than the rest of the cohort. Dobyns et al. ${ }^{[8]}$ similarly reported that patients with more severe lung

Table 3. Length of treatment and duration of admission of patients treated with iNO

\begin{tabular}{|c|c|c|c|}
\hline & $\begin{array}{l}\text { Length of treatment, } \\
\text { median (IQR) }\end{array}$ & $\begin{array}{l}\text { ICU days, } \\
\text { median (IQR) }\end{array}$ & $\begin{array}{l}\text { Hospital days, } \\
\text { median (IQR) }\end{array}$ \\
\hline Cohort & $3(2-4)$ & $15(9-23)$ & $29(19-50)$ \\
\hline PHT post-cardiac surgery & $3(2-4)$ & $16(9-22)$ & $30(20-51)$ \\
\hline ARDS & $3(2-4)$ & $13(9-23)$ & $28(16-44)$ \\
\hline Adenovirus & $3(2-6)$ & $20(11-26)$ & $30(21-52)$ \\
\hline Adenovirus: survivors & $4(2-6)$ & $23(20.5-28.5)$ & $48(29-60.5)$ \\
\hline PPHN & $3.5(2.3-4.8)$ & $23(6-50.5)$ & $38(13-61)$ \\
\hline Underweight for age & $3(2-4)$ & $16(9-24.5)$ & $33(20-56.5)$ \\
\hline HIV & $5(2.7-5)$ & $12(4-17)$ & $24(13-33.5)$ \\
\hline Premature at birth & $3(2.3-3.75)$ & $18(10-26)$ & $34(18-52)$ \\
\hline TB & $3.5(2-9)$ & $23(14.5-36.5)$ & $38.5(29-49.5)$ \\
\hline Sepsis & $3(2-5)$ & $18(11-25)$ & $32(22-52)$ \\
\hline
\end{tabular}


Table 4. Annual expenditure on iNO

\begin{tabular}{ll}
\hline Year & Cost (ZAR) \\
\hline 2011 & 345563 \\
2012 & 229824 \\
2013 & 178558 \\
2014 & 352676 \\
2015 & 334755 \\
Total over 5 years & 1441376 \\
iNO = inhaled nitric oxide. &
\end{tabular}

disease, those with a high oxygenation index (OI) on starting treatment, and those that are immunocompromised respond better to treatment. This may be related to the role of iNO in modulating inflammation. ${ }^{[2]}$ Inhaled NO is used universally in our unit as rescue therapy in patients with ARDS that are failing treatment with ventilation alone. There is however some evidence showing that early initiation of treatment, irrespective of disease severity improves outcomes. ${ }^{[19]}$

The literature shows that iNO effects only a transient improvement in oxygenation..$^{[3-9]}$ Part of the rationale behind continued use is that this temporary improvement in oxygenation may afford time for other therapies to be instituted or time for recovery of the underlying condition itself. Notwithstanding, a decrease in mortality following treatment with iNO has not been shown. Possible reasons are that these patients often have multi-organ dysfunction that is not improved by iNO, and because a response to treatment with improved oxygenation does not necessarily indicate an improvement in lung parenchymal disease.

The rationale for initiating iNO therapy in patients' post-cardiac surgery is to decrease PVR in an attempt to ameliorate PHTCs. The lack of significant benefit with treatment, however, suggests that there are multiple factors contributing to the development of PHTCs, such as the ability of the right ventricle to handle an increase in PVR rather than the absolute increase in PVR itself.

Mortality in the entire cohort treated with iNO was $31 \%$, and $53 \%$ in those with ARDS. This is higher than the reported mortality rate by Medjo et al., ${ }^{[10]}$ Day et al., ${ }^{[7]}$ Dobyns et al. ${ }^{[8]}$ and Dowell ${ }^{[13]}$ of $44 \%, 46 \%$, $43 \%$ and $34 \%$, respectively. Mortality was $63 \%$ in those with adenoviral disease, despite $71 \%$ of this group responding to treatment. We found no difference in mortality rate in responders v. non-responders. This is in line with the findings of Dowell ${ }^{[13]}$ and Lundin et al. ${ }^{[20]}$

The cost of treating the cohort with iNO was ZAR1 441376 during the period being reviewed, which is a considerable strain on a resourcelimited setting. Gupta et al. ${ }^{[12]}$ was the first to show worse outcomes in terms of costs and morbidity in patients treated with iNO. We also report longer lengths of admission in patients with ARDS and those with $\mathrm{TB}$. When the group with adenoviral infection is divided into those who survived to discharge and those who did not, we see markedly longer lengths of admission in the survivors, compounding the costs incurred in treating these patients, although these findings were not statistically significant. Patients with sepsis were associated with longer lengths of treatment ( $p=0.02)$, longer lengths of ICU admission $(p=0.00)$, and longer lengths of hospital admission $(p=0.00)$.

\section{Study limitation}

The present study was a retrospective review without a control group, and many patient's files and data points were missing. What we did find was that practice was not standardised in our unit owing to a lack of protocols guiding the indications for use, dosage, weaning and discontinuation of treatment. Echocardiography, which is the standard non-invasive investigation of choice in diagnosing PHT recommended by the AHA and European Consensus guidelines ${ }^{[11,17,18]}$ was not routinely used. The electronic cardiology database was accessed in an attempt to gather data on pulmonary pressures before and after treatment. This was abandoned because of missing data for most patients.

A positive response to treatment was judged by the attending clinician and was usually based on an increase in oxygen saturation alone. There was no defined increment in saturation that was used to denote a response. Oxygenation indices were not calculated in any of the patients that were treated, and it was not possible to calculate these retrospectively due to missing patient records and missing data points. Most studies defined a positive response as improvement in the oxygenation index (OI) of $20 \mathrm{E}$ from baseline. ${ }^{[8,13,16,17,20]}$ Response to treatment as judged by an increase in oxygen saturation alone may have been confounded by adjunctive measures or therapies such as prone positioning, high frequency oscillatory ventilation, neuromuscular blockade or changes in ventilator settings. Response to treatment or lack thereof was not well documented in patients' medical records. We often use iNO in our unit as rescue therapy in patients with PHTCs. However, iNO is used prophylactically in an attempt to prevent the onset of PHTCs in all the published literature. ${ }^{[15,16,21]}$ We did not have access to data regarding the number of PHTCs in our cohort. Sildenafil is recommended for use in all patients when weaning treatment with iNO. ${ }^{[18]}$ Only $47 \%$ of our cohort was treated concomitantly with sildenafil when weaning treatment with iNO.

\section{Conclusions}

We report that $64 \%$ of patients treated with iNO responded to treatment. Response to treatment was, however, poorly defined and not well documented in the medical records. Despite this response to treatment, mortality was high, particularly in those with ARDS and adenoviral infection. Length of hospital and PICU admission were also longer in these patients. Further studies in patients with ARDS stratified for underlying pathology are required. Moreover, we need further studies to look at long-term outcomes and cost effectiveness in resource-limited settings to justify the ongoing use of this expensive treatment.

In patients with PHT following cardiac surgery, iNO as rescue v. prophylactic therapy needs to be evaluated in good quality RCTs. It is also not clear whether the type of underlying cardiac condition is a predictor of response to iNO.

Overall, practice was not standardised in our unit and the cost of using this treatment is high.

The continued use of this treatment should be protocol driven, with clear criteria for indications for use, dosage and weaning, discontinuation, and determining a response to treatment. This should include the use of the OI and echocardiographic evidence of PHT. ${ }^{[1,17,18]}$

Declaration. This study was done in partial fulfil of the requirements of an MMed (Paed) degree.

Acknowledgements. None.

Author contributions. Equal contributions.

Funding. None.

Conflicts of interest. None. 
1. Gebistorf F, Karam O, Wetterslev J, Afshari A. Inhaled nitric oxide for acute respiratory distress syndrome (ARDS) in children and adults. Cochrane Database Syst Rev 2016(6):CD002787. https://doi.org/10.1002/14651858.cd002787.pub3

2. Kinsella JP, Abman SH. Inhaled nitric oxide therapy in children. Paediatric Respir Rev 2005;6(3):190-198. https://doi.10.1016/j.prrv.2005.06.02

3. Steinhorn RH. Therapeutic approaches using nitric oxide in infants and children. Free Radical Bio Med 2011;51(5):1027-1034. https://doi.org/10.1016/j.freeradbiomed.2011.01.006

4. Adhikari NK, Dellinger RP, Lundin S, et al. Inhaled nitric oxide does not reduce mortality in patients with acute respiratory distress syndrome regardless of severity: Systematic review and meta-analysis. Crit Care Med 2014;42(2):404-412. https://doi.org/10.1097/ccm.0b013e3182a27909

5. Afshari A, Brok J, Moller AM, Wetterslev J. Inhaled nitric oxide for acute respiratory dstres syndrome and acute lung injury in adults and children: A systematic review with meta-analysi and trial sequential analysis. Anaesth Analg 2011;112(6):1411-1421. https://doi.org/10.1213/ ane.0b013e31820bd185

6. Adhikari NK, Burns KE, Friedrich JO, Granton JT, Cook DJ, Meade MO. Effect of nitric oxide on oxygenation and mortality in acute lung injury: Systematic review and meta-analysis. BM] 2007;334(7597):779. https://doi.org/10.1136/bmj.39139.716794.55

7. Day RW, Allen EM, Witte MK. A randomised, controlled study of the 1-hour and 24-hour effects of inhaled nitric oxide therapy in children with acute hypoxaemic respiratory failure. Chest 1997;112(5):1324-1331. https://doi.org/10.1378/chest.112.5.1324

8. Dobyns EL, Cornfield DN, Anas NG, et al. Multicentre randomised controlled trial of the effects of inhaled nitric oxide therapy on gas exchange in children with acute hypoxaemic respiratory failure. J Pediatr 1999;134(4):406-412. https://doi.org/10.1016/s0022-3476(99)70196-4

9. Bronicki RA, Fortenberry J, Schreiber M, Checchia PA, Anas NG. Multicent randomised controlled trial of inhaled nitric oxide for paediatric acute respiratory distress syndrome. J Pediat 2015;166(2):365-369. https://doi.org/10.1016/j.jpeds.2014.10.011

10. Medjo B, Atanaskovic-Markovic M, Nikolic D, Cuturilo G, Djukic S. Inhaled nitric oxide therapy for acute respiratory distress syndrome in children. Indian Pediatr 2012;49(7):573-576.

11. Hansmann G, Apitz C. Treatment of children with pulmonary hypertension. Expert consensu statement on the diagnosis and treatment of paediatric pulmonary hypertension. The Europea Paediatric Pulmonary Vascular Disease Network, endorsed by ISHLT and DGPK. Heart 2016;102(2):67-85. https://doi.org/10.1136/heartjnl-2015-309103

12. Gupta P, Richardson T, Hall M, et al. Effect of inhaled nitric oxide on outcomes in children with acute lung injury: Propensity matched analysis from a linked database. Crit Care Med 2016;44(10):1901-1909. https://doi.org/10.1097/ccm.0000000000001837
13. Dowell JC, Thomas NJ, Yehya N. Association of response to inhaled nitric oxide and duration of mechanical ventilation in paediatric acute respiratory distress syndrome. Paediatr Crit Care Med 2017;18(11):1019-1026. https://doi.org/10.1097/pcc.0000000000001305

14. Bizzarro M, Gross I, Barbosa FT. Inhaled nitric oxide for the postoperative management of pulmonary hypertension in infants and children with congenital heart disease. Cochran Database Syst Rev 2014(7):CD005055. https://doi.org/10.1002/14651858.cd005055.pub3

15. Miller OI, Tang SF, Keech A, Pigott NB, Beller E, Celermajer DS. Inhaled nitric oxide and prevention of pulmonary hypertension after congenital heart surgery: A randomised doubleblind study. Lancet 2000;356(9240):1464-1469. https://doi.org/10.1016/s0140-6736(00)02869-5

16. Day RW, Hawkins JA, McGough EC, Crezee KL, Orsmond GS. Randomised controlled study of inhaled nitric oxide after operation for congenital heart disease. Ann Thorac Surg 2000;69(6):1907-1912. https://doi.org/10.1016/s0003-4975(00)01312-6

17. Abman SH, Hansmann G, Archer SL, et al. Pediatric pulmonary hypertension: Guidelines from the American Heart Association and American Thoracic Society. Circulation 2015;132(21):2037-2099. https://doi.org/10.1161/cir.0000000000000329

18. Kaestner M, Schranz D, Warnecke G, Apitz C, Hansmann G, Miera O. Pulmonary hypertension in the intensive care unit. Expert consensus statement on the diagnosis and treatment of paediatric pulmonary hypertension. The European Paediatric Pulmonary Vascular Disease Network, endorsed by ISHLT and DGPK. Heart 2016;10(2):57-66. https://doi.org/10.1136/ heartjnl-2015-307774

19. Fioretto JR, de Moraes MA, Bonatto RC, Ricchetti SM, Carpi MF. Acute and sustained effects of early administration of inhaled nitric oxide to children with acute respiratory distress syndrome. Paediatr Crit Care Med 2004;5(5):469-474. https://doi.org/10.1097/01 pcc.0000137986.83738.d 7

20. Lundin S, Mang H, Smithies M, Stenqvist O, Frostell C. Inhalation of nitric oxide in acute ung injury: Results of a European multi-centre study. Intensive Care Med 1999;25(9):911-919. https://doi.org/10.1007/s001340050982

21. Morris K, Beghetti M, Petros A, Adatia I, Bohn D. Comparison of hyperventilation and inhaled nitric oxide for pulmonary hypertension after repair of congenital heart disease. Crit Care Med 2000;28(8):2974-2978. https://doi.org/10.1097/00003246-200008000-00048

Accepted 16 March 2021 